Governments of many countries to combat, for example, hookworm, malaria and yellow fever; and at the same time to institute centres of public health, to promote nursing education, and to develop medical schools. An instance of significant medical benefaction has been the support in large part of the Peking Union Medical College in China ; again, the London School of Hygiene and Tropical Medicine was built and equipped by a gift of half a million sterling. The recent larger gifts of the Foundation in England have been mainly for other than medical purposes, such as funds for the University of London site in Bloomsbury, the grant to the London School of Economics, the grant to Cambridge to include the new library, and about three fifths of the sum needed for the Bodleian extension at Oxford, the foundation stone of which will be laid on June 25 by Queen Mary. The total of these benefactions amounts to $£ 1,872,000$.

In 1929, the trustees of the Foundation, again acting with the free hand which the policy of Rockefeller accorded them, absorbed a fourth trust set up some years earlier by the founder, the Laura Spelman Rockefeller Memorial, named after his wife and dedicated to the aid of the social sciences and to the welfare of children. The work of the whole now comprehends not only the international health division of the Foundation and the social sciences division of the Memorial, but also divisions for medical sciences, natural sciences and the humanities.

A characteristic of the Rockefeller philanthropies is that their practice comes from time to time under systematic review and revision by the trustees. This is done in order to meet the altering social needs of given countries. Vigilant attention to system, at home in the General Education Board and in the Institute for Medical Research, abroad in the Foundation whose work now encompasses the world, is the heritage of the business genius of John D. Rockefeller.

\title{
Regularities and Irregularities in the Ionosphere
}

$\mathrm{P}^{\mathrm{R}}$ ROF. E. V. APPLETON, in the Bakerian Lecture before the Royal Society, delivered on June 10, surveyed the information derived from radio sounding of the ionized regions of the upper atmosphere and compared the results with those derived from a theory of simple layer formation by solar ionizing radiation, travelling rectilinearly and attenuated according to a massabsorption law. Such a formation is called a 'simple region'. The comparisons are concerned chiefly with the variation of maximum electron content $N_{\mathrm{m}}$ with solar radiation angle of incidence $\chi$, with the total conductivity for direct currents such as determines the quiet-day magnetic variations and with the highfrequency absorption of radio waves traversing such a region, the two latter quantities being, to some extent, related.

For the lower Region $E$, where the daytime recombination is sufficiently rapid to maintain the electron concentration close to the equilibrium value, recombination substantially balancing ionization, the value of $N_{\mathrm{m}}$ is found to vary, experimentally and theoretically, as $(\cos \chi)^{1 / 2}$. The high-frequency absorption for radio transmission, on the other hand, varies theoretically as $(\cos \chi)^{3 / 2}$. Here fair agreement is found with the experimental seasonal noon variation when the sun is high, as in summer, but the winter values show disagreement, the absorption being then greater than simple theory predicts. For the case of the diurnal variation of absorption during a summer day, Best and Ratcliffe, working at Cambridge, have found good agreement with the theoretical relation.

No evidence is found suggesting the existence of permanent highly reflecting regions in the lower or middle atmosphere.

From the high-frequency absorption and other data, the transverse direct-current electron conductivity of a simple region is estimated and found to be less than that calculated by Schuster and Chapman from the magnitude of the magnetic variations, even after allowing for the revised estimates of upper-atmospheric motions recently made by Pekeris. This difficulty is met by the assumption of the existence of quantities of positive and negative ions in the lower part of the ionosphere, undetectable by radio methods, but which contribute to the conductivity for steady electromotive forces. From this assumption follows an explanation of the high effective electron recombination coefficient in the lower region, where the value is high because of the large number of positive ions. There is a marked difference between the day and night values of the effective electron recombination coefficients in Region $E$.

The identification of the lower regions of the ionosphere with the levels at which flow the currents affecting terrestrial magnetism is supported by evidence from systematic measurements of the value of $N_{\max }$, for Regions $E, F_{1}$ and $F_{2}$, which show that the ionization in Regions $E$ and $F_{1}$ varies by about 50 per cent over the sunspot cycle, as does the magnetic variation. The variation of Region $F_{2}$ ionization, on the other hand, exhibits no parallelism with the magnetic changes, either in respect of seasonal or sunspot cycle trends, indicating that the magnetic currents do not flow at high levels.

The two types of ionospheric abnormalities, the one associated with bright solar eruptions and the other with terrestrial magnetic storms, were compared and discussed.

An estimate of the actual heights reached by radio waves at the level of reflection leads, in turn, to an estimate of the proportional variation of air density with height for the ionized component. From the fact that Region $F$ is found to be some four or five times as thick as Region $E$, it follows that the local 'scale-height', $H$, in Region $F$ is correspondingly greater than in Region $E$. The atmosphere therefore extends to much greater heights than are estimated from the simple exponential decrease of pressure with height found in the lower atmosphere. Such an extension of the ionizable gases to great heights indicates either that the temperature at $300 \mathrm{~km}$. is higher than at lower levels, or else that it is there composed largely of a light gas such as helium. Difficulties in the way of the acceptance of the latter alternative are discussed. 